\title{
Rounding of aggregates of biological cells: Experiments and simulations
}

\author{
José C.M. Mombach ${ }^{1}$, Damien Robert ${ }^{2}$, François Graner $^{3}$, \\ Germain Gillet ${ }^{4}$, Gilberto L. Thomas ${ }^{5}$, Marco Idiart ${ }^{5}$, \\ Jean-Paul Rieu ${ }^{2}$
}

(1) Laboratório de Bioinformática e Biologia Computacional Universidade do Vale do Rio dos Sinos, Av. Unisinos, 950 93022-000 São Leopoldo, RS, Brazil.

(2) Laboratoire de Physique de la Matière Condensée et Nanostructures, UMR5586 CNRS and University Lyon 1, 43 boulevard du 11 Novembre, 69623 Villeurbanne, France.

(3) Laboratoire de Spectrometrie Physique, UMR5588 CNRS and Université Grenoble I BP 87, 38402 St. Martin D'Heres Cedex, France.

(4) Institut de Biologie et Chimie des Protéines, CNRS and University Lyon 1, 7 passage du Vercors, 69367 Lyon Cedex 07, France.

(5) Instituto de Física, Universidade Federal do Rio Grande do Sul, Av. Bento Gonçalves, 9500 91501-970 Porto Alegre, RS, Brazil.

\begin{abstract}
The influence of surface tension and size on rounding of cell aggregates are studied using chick embryonic cells and numerical simulations based on the cellular Potts model. Our results show exponential relaxation in both cases as verified in previous studies using 2D Hydra cell aggregates. The relaxation time decreases with higher surface tension as expected from hydrodynamics laws. However, it increases faster than linearly with aggregate size. The results provide an additional support to the validity of the cellular Potts model for non-equilibrium situations and indicate that aggregate shape relaxation is not governed by the hydrodynamics of viscous liquids.
\end{abstract}

\section{Introduction}

It is now well established that certain living tissues behave as viscoelastic fluids [1. Classical experiments on the relaxation of embryonic aggregates subjected 
to mechanical deformations, either by centrifugation [2] or by compression [3], demonstrated that tissues relax as elastic materials on short time scales and as viscous liquids on long time scales. The relaxation of compressed cellular aggregates was analyzed using a Kelvin model of viscoelasticity [3. As stated by the authors, this model may represent a strong oversimplification of the tissue rheology but it gives an estimation of the tissue elastic and viscous parameters [3]. The fusion or the rounding up of cell aggregates are similar to the coalescence or rounding up of liquid droplets. Gordon et al. showed that these processes are driven by tissue surface tensions $\sigma$ and opposed by tissue viscosity $\eta$ making a formal analogy with the rheology of viscous liquids. They obtained first estimates of the ratio $\eta / \sigma$ for chicken embryonic cells [4]. However, the authors gave only orders of magnitude of this ratio as they performed only preliminary experiments, presumably because it was difficult to obtain suspended three dimensional aggregates which were fixed over long times (rotation or flattening on a surface are undesirable). Later, the surface tensions of different chicken embryonic tissues have been measured using a parallel plate compression apparatus [5, 6].

In previous investigations one of us showed that a two dimensional (2D) assembly of Hydra cells behaves like a fluid: (i) cells exhibit collective [7 8] or random [9] motion, (ii) the hydrodynamical laws also apply for the rounding-up of initially elliptical aggregates $[8$. The shape relaxation was analyzed using the 2D hydrodynamical equations derived by Mann et al. for polymer monolayers in the case of dissipation dominated by surface viscosity [10. This method allowed an estimation $\eta / \sigma \simeq 0.5 \mathrm{~min} / \mu \mathrm{m}$ although $\sigma$ was not measured independently. Compared to the relaxation analysis of free 3D aggregates, 2D aggregates offer some advantages: they are not moving, nor rotating or flattening on a nearby surface. The $2 \mathrm{D}$ system also allows to track simultaneously cell motion and a direct comparison with 2D simulations.

In this paper, we summarize the main results obtained previously on $2 \mathrm{D}$ Hydra aggregates []. We also present current experiments on the rounding of 3D aggregates of chicken embryonic cells (neural cells from the retina) and simulations of rounding of 2D aggregates using the cellular Potts model. Because of its flexibility and simplicity of implementation, Potts model has become a common technique for cell level simulation of biological tissues. It describes effectively the global features of tissue rearrangement experiments, including cell sorting [11 and even the cell movements during the entire life cycle of the slime mould Dictyostelium discoideum [12]. In this study, we show that Potts model effectively captures the exponential relaxation dynamics of aggregate rounding. However, both new experiments and simulations show a deviation from the hydrodynamics of viscous liquids. 


\section{Results}

\subsection{Rounding of 2D Hydra aggregates}

Hydra aggregates were prepared according to previously reported methods [7] with $25 \mu \mathrm{m}$ spacers (Fig. 1A). A sequence of events in a pure endodermal aggregate initially elliptical is displayed in Figs. 1B-C. Rounding up is completed after $6 \mathrm{~h}$. We studied also the rounding of mixed and pure ectodermal aggregates [8. Images were digitized at intervals of $5 \sim 10$ min and analyzed on a computer using the software NIH Image [13. The deformation parameter $d=\frac{M}{m}-1$ was used to describe the aggregate shape, where $M$ and $m$ are the major and minor axis of the fitted ellipse. $d$ is zero for a perfect circle and increases for elongated aggregates.

Fig. 1D shows a log-linear plot of the time dependence of the $d$ for the different $2 \mathrm{D}$ aggregates investigated. For each aggregate, $d$ decreases exponentially with time. Such exponential behavior is expected theoretically in 2D from the hydrodynamics laws in case of viscosity dominated by the surface viscosity and rounding driven by line tension [10. In case of 2D aggregates confined between two glass slides, if the cell/glass friction may be neglected compared to cell/cell friction, the relaxation time is given by relation:

$$
T_{c}=\frac{\eta}{\sigma} R
$$

where $R$ is the radius of the rounded aggregate (final time), $\eta$ is a bulk surface viscosity, and $\sigma$ the tissue/external medium surface tension [8]. The typical time scale of rounding depends thus on the ratio between the surface tension and viscosity. The aggregates round to decrease superficial tension, while viscosity acts in the sense of delaying the rounding. Fig. 1E shows that for each aggregate type, $T_{c}$ is increasing with $R$ as expected from the hydrodynamical law Eq. 1 The ratio $\eta / \sigma=0.5 \pm 0.2 \mathrm{~min} / \mu \mathrm{m}$ which gives $\eta=3 \times 10^{4} \mathrm{~Pa} . \mathrm{s}(1 \mathrm{~Pa} . \mathrm{s}=10$ Poise) using $\sigma=1 \mathrm{mN} / \mathrm{m}$ as a typical value of tissue surface tension [3, 5, 6]. This is $10^{7}$ times higher than the water viscosity.

\subsection{Rounding of $3 \mathrm{D}$ chicken embryonic neural retina ag- gregates}

We performed relaxation experiments on chicken embryonic tissues which have been already characterized by surface tensiometry [3, 5]. Neural cell tissues were obtained from the retina of 9 day old chicken embryos. After dissection and trypsinization, cells were reaggregated by shaking them slowly in 12-well plates (200 r.p.m.) containing culture medium (BME culture medium with $10 \%$ fetal Calf Serum [14) at $37^{\circ} \mathrm{C}$ during 16 hours. We could not obtain proper 2D chicken aggregates for relaxation experiments. We used instead noncompressed 3D aggregates, just floating near a bottom slide glass (Fig. 2A). These aggregates are sufficiently stable for shape analysis, they do not move or rotate significantly and do not spread on the bottom slide glass. Figures 2B-C 
show the shape of a 9-day old 3D aggregate between initial and final time (24h). The time dependence of the deformation parameter of these aggregates is well exponential as expected from hydrodynamics with a relaxation time $T_{c}$ of about 1 day (Fig. 2D). In case of rounding of $3 \mathrm{D}$ ellipsoid droplets, $T_{c}$ is given by a slightly different relation than the $2 \mathrm{D}$ equation 10 4]:

$$
T_{c}=\beta \frac{\eta}{\sigma} R
$$

where the constant $\beta \sim 0.95$ in case of aggregate internal viscosity much larger than external medium viscosity [4, and $R$ is the radius of the rounded 3D aggregate.

At a first sight, $T_{c}$ seems to increase linearly with aggregate radius. The doted line in Fig. 2E corresponds to such a linear fit with Eq. 2 (correlation coefficient, 0.880 ). The ratio $\eta / \sigma$ is $16.8 \pm 4.5 \mathrm{~min} / \mu \mathrm{m}$. The surface tension of neural aggregates dissected at 6 days and maintained in culture for various time periods was measured with the compression plate apparatus $[6]$. The value increases with continued incubation for about a day (between 6 and 7 days), then stabilizes for a time period of about 2 days at $\sigma=1.6 \pm 0.6 \mathrm{mN} / \mathrm{m}$ and increases again between 8 and 9 days to about $\sigma=4.0 \pm 1.0 \mathrm{mN} / \mathrm{m}$. Taking this latter value, we obtain the following neural tissue viscosity value at 9 days: $\eta \simeq(4 \pm 2) \times 10^{6} \mathrm{~Pa}$.s. It is much higher than in Hydra aggregates. This is not surprising. First, because the kinetics of rounding is much slower in chicken embryonic neural aggregates. Also because cell packing is much higher in these 3D aggregates (Fig. 2B-C) than in the 2D Hydra aggregates (Fig. 1B-C). It is likely that tissue surface tension and tissue viscosity depend both on cell/cell adhesion [8] and that adhesion is higher for 3D chicken embryonic aggregates than 2D Hydra aggregate.

However, $T_{c}$ is probably increasing faster than linearly, i.e., a power law $\sim R^{1.52}$ fits better the data (correlation coefficient, 0.939). Although there is large dispersion of the experimental points which is often inherent to such a biological system, the visual impression is also better than with linear fit (Fig. $2 \mathrm{E})$. Of course, these data should be confirmed but they seem to indicate that 3D chick embryonic cell aggregates do not behave as viscous liquids. The relaxation of initially elongated aggregates toward a circular shape is clearly exponential but relaxation time is not proportional to the aggregate size.

\subsection{Simulations}

The model is based on the cellular Potts model [1] and is defined as follows: at each site $(i, j)$ of a square lattice with dimensions $L \times L$ we attribute a spin that may assume any integer value. The set of all sites with equal spin $S$ defines a cell, labeled by S.

Adhesion between cells originates in the interaction between each spin and its 20 surrounding neighbors (up to fourth neighbors to avoid pinning to the square lattice [15]). The lowest interaction energy, here taken as zero, happens between

equal spins, simulating the absence of surface tension between sites belonging 
to the same cell. Energy between different cells is taken to be positive and when different types of cells are considered, the interaction energy may also be different, depending on the types involved in the interaction. The intensity of each possible interaction must be specified. Hence, for example, for a system made of an aggregate of cells of two types only and medium we must define three parameters. The result is a proportional to the shared interface length, with a proportionality constant depending on the neighboring cell types to reflect differential adhesion strengths.

Cell resistance to compression is modeled by an energy term proportional to the square deviation of cell area from its target area $A_{T}(S)$. The proportionality parameter $\lambda$ plays the role of a Lagrange multiplier for this constraint and regulates cell compressibility. The complete energy used in the Monte Carlo protocol is

$$
H=\sum_{i j} \sum_{i^{\prime} j^{\prime}} E_{S_{i j} S_{i^{\prime} j^{\prime}}}\left(1-\delta_{S_{i j}, S_{i^{\prime} j^{\prime}}}\right)+\lambda \sum_{S}\left[a(S)-A_{T}(S)\right]^{2},
$$

where $a(S)$ is the area of cell $S, S_{i j}$ is the spin at site $(i, j)$, and $E_{S_{i j}, S_{i^{\prime} j^{\prime}}}$ is the interaction energy between neighboring sites labeled by $S_{i j}$ and $S_{i^{\prime} j^{\prime}}$. If $S_{i j}=S_{i^{\prime} j^{\prime}}$ then $E_{S_{i j}, S_{i^{\prime} j^{\prime}}}=0$ since in this case both sites belong to the same cell.

The aggregate surface tension $(\sigma$, at $T=0)$ derives from the coupling energies in Eq. (3) by [1]

$$
\sigma=E_{c M}-\frac{E_{c c}}{2},
$$

where $E_{c M}$ and $E_{c c}$ are the adhesion energies per unit of interface between cells and medium and between cells, respectively.

We implement the simulations as follows. We randomly choose a lattice site $(i, j)$ and one of its eight surrounding neighbors. We then propose that the site assumes the neighboring spin value $S^{\prime}$ and accept with probability $P$ :

$$
P\left(S \rightarrow S^{\prime}\right)= \begin{cases}\exp (-\Delta H / T) & \text { if } \Delta H>0, \\ 1 & \text { if } \Delta H \leq 0,\end{cases}
$$

where $\Delta H$ is the variation in energy produced by the change of spin values. A Monte Carlo Step (MCS), is defined as $L^{2}$ exchange attempts, the total number of sites in the lattice. For sake of simplicity, we use the MCS as the unit of "time" in what follows. In fact, the random sampling of spins, and the immediate dissipation of their energy (perfectly local, both in time and space) imply that the correspondence with real time is not straightforward [16].

In this model the simulation temperature $T$ relates to active cell membrane motion. Higher $T$ implies higher membrane activity and vice-versa. $T$ simulates membrane fluctuations driven by the cytoskeleton in real cells and should not be confused with much smaller thermal fluctuations [11.

Aggregate rounding was investigated using as initial states elliptical aggregates with different numbers of cells: 544, 953, 1642, 2204, and 3233 cells. Cells 
areas have approximately 100 lattice sites and cell-medium surface tensions have values: $4.5,5.5,6.5,7.5,8.5,9.5$. To generate these surface tensions we keep constant the interaction energy between cells, $E_{c c}=1$, and vary the energy between cells and medium, $E_{c M}=5,6,7,8,9$, and 10. The simulation temperature used is 10 and $\lambda=1$. For aggregates with 1642 cells we simulated all of the above surface tensions, however for other aggregate sizes we studied only the three lower values of surface tension. In Figs. 3A and 3B we show, respectively, the initial and final state (complete rounding-up) of the simulated aggregate with 544 cells.

We calculate the deformation in the simulated aggregates using the ratio of the eigenvalues of the two dimensional variance of mass distribution of the aggregate that is defined as

$$
\left(\begin{array}{cc}
\left\langle x^{2}\right\rangle-\langle x\rangle^{2} & \langle x y\rangle-\langle x\rangle\langle y\rangle \\
\langle x y\rangle-\langle x\rangle\langle y\rangle & \left\langle y^{2}\right\rangle-\langle y\rangle^{2}
\end{array}\right)
$$

where $x$ and $y$ are the coordinates of all lattice sites belonging to cells in the aggregate. As the aggregate rounds, the ratio between the two eigenvalues of the matrix above, $e_{1}$ and $e_{2}$ goes to 1 . In analogy with experimental studies, we define aggregate deformation using the ratio between the larger and lower eigenvalues of the matrix minus one: $d=e_{1} / e_{2}-1,\left(e_{1}>e_{2}\right)$. This measure of the deformation differs from that used in the experiments but should yield the same exponential decay.

In Fig. 4 we plot $d$ as function of time for aggregates with 1642 cells for surface tensions $4.5,6.5,8.5$, and 9.5. The relaxation is clearly exponential in all cases. Fig. 5 shows $T_{c}$ as a function of $\sigma^{-1}$ for simulated aggregates with 1642 cells and surface tensions 4.5, 5.5, 6.5, 7.5, 8.5, and 9.5. A statistical analysis of the fit in the figure supports an intercept of the line with the vertical axis at the origin ( $p$-value of linear coefficient $=0.8$ ), consistent with Eq. 1 According to Eq. 1 the slope of the fit is the $\mathrm{R} \times$ viscosity, however simulations predict an exponent higher than 1 from a $\log -\log$ plot of $T_{c}$ vs. aggregate radius. As we can see in Fig. 6, we determined that the slope for simulation is about 2.5 and for chicken data is about 1.5 (see also fit in Fig. 2). Simulation exponent seems robust to variations in temperature and surface tension. In both cases the results suggest a deviation from hydrodynamics.

\section{Conclusions}

We have presented new experimental results on the relaxation of biological cell aggregates (chick embryonic neural retina aggregates). Our motivation was to measure the equivalent viscosity of this tissue. Such measurements are important to characterize cell adhesiveness and to provide model parameters for theoretical or numerical models of collective motion [17. The relaxation of 3D aggregates is exponential, however, contrary to 2D aggregates of Hydra cells [8], relaxation time seems to increase faster than linearly with aggregate size. We are currently working on the verification of this experimental dependence 
in case of $3 \mathrm{D}$ aggregates. To this end, we need to improve the experimental environment $\left(\mathrm{CO}_{2}\right.$ and $\mathrm{O}_{2}$ control) in order to increase the observation time to a few days and be able to study larger aggregates. It should be also noted that 2D results suffer large errors. However if this dependence is confirmed, the reason why $2 \mathrm{D}$ Hydra aggregates behave as viscous fluid but not the $3 \mathrm{D}$ chick aggregates is not completely clear. The answer may arise from cell trajectory studies. In case of $2 \mathrm{D}$ hydra aggregates, all cells participated to rounding, motion was very collective [8]. We have not performed a similar study yet but it seems that in larger 3D aggregates, intense cell movements occur mainly in the aggregate periphery in a band of a few cell diameter while in the core of the aggregate cells

are less active. Assuming nevertheless a fluid behavior, we obtain the following estimate for the tissue viscosity of 9 day old neural retina, $\eta \simeq(4 \pm 2) \times 10^{6}$ Pa.s.

A simple Potts model simulation of the phenomena captures the exponential decay and indicates a superlinear dependence of relaxation time with aggregate size as observed in experiments with chicken cells. The simulation exponent, however does not agree with experiments but a possible explanation for the difference may come from cell trajectory studies. 3D simulations might also provide useful quantitative comparisons with 3D aggregates.

\section{Acknowledgments}

We acknowledge useful discussions with Rita M. C. de Almeida, and M. Aubouy. This work is a brazilian-french collaboration supported by Capes and Cofecub agencies project $n^{\circ} 414 / 03$. J.C.M. Mombach acknowledges the partial support of HP Brazil R\&D. J.P. Rieu and G. Gillet acknowledge the partial support of french C.N.R.S. (ACI Dynamique et Réactivité des Assemblages Biologiques).

\section{References}

[1] D. A. Beysens, G. Forgacs, J. A. Glazier, Proc. Natl. Acad. Sci. USA 97, (2000) 9467.

[2] H. M. Phillips, M. S. Steinberg, J. Cell Sci. 30, (1978) 1.

[3] G. Forgacs, R. A. Foty, Y. Shafrir, M. S. Steinberg, Biophys. J. 74, (1998) 2227.

[4] R. Gordon, N. S. Goel, M. S. Steinberg, L. L. Wiseman, J. Theor. Biol. 37, (1972) 43 .

[5] R. A. Foty, G. Forgacs, C. M. Pfleger, M. S. Steinberg, Phys. Rev. Lett. 72, 2298 (1994).

[6] R. A. Foty, C. M. Pfleger, G. Forgacs, M. S. Steinberg, Development 122, (1996) 1611. 
[7] J.-P. Rieu, N. Kataoka, Y. Sawada, Phys. Rev. E 57, (1998) 924.

[8] J.-P. Rieu, Y. Sawada, Eur. Phys. J. B. 27, (2002) 167.

[9] J.-P. Rieu, A. Upadhyaya, J. A. Glazier, N. B. Ouchi, Y. Sawada, Biophys. J. 79, (2000) 1903.

[10] E. K. Mann, S. Hénon, D. Langevin, J. Meunier, L. Léger, Phys. Rev. E 51, (1995) 5708.

[11] F. Graner, J. A. Glazier, Phys. Rev. Lett. 69, (1992) 2013; J. A. Glazier, F. Graner, Phys. Rev. E 47, (1993) 2128.

[12] A. F. Marée and P. Hogeweg, Proc. Natl. Acad. Sci. USA 98, (2001) 3879. H. Levine, I. Aranson, L. Tsimring and T. V. Truong, Proc. Natl. Acad. Sci. USA 93, (1996) 638. Y. Jiang, H. Levine and J. A. Glazier, Biophys. J. 75, (1998) 2615. C. Van Oss, A. V. Panfilov, P. Hogeweg, F. Siegert, C. J. Weijer, J. Theor. Biol. 181, (1996) 203.

[13] NIH Image is a public domain program developed at the U.S. National Institutes of Health and available on the Internet at http://rsb.info.nih.gov/nih-image/.

[14] G. Gillet, D. Michel, P. Crisanti, M. Guerin,Y. Herault, B. Pessac, G. Calothy, G. Brun, M. Volovitch. Oncogene 8, (1993) 565.

[15] E. Holm, J. A. Glazier, D. J. Srolovitz, and G. S. Grest, Phys. Rev. A 43, (1991) 2262.

[16] K. Binder, D.W. Heermann, Monte Carlo Simulation in Statistical Physics, Springer, Berlin, third edition, 1997.

[17] E. Palsson, H. G. Othmer, Proc. Nat. Acad. Sci. USA 97, (2000) 10448. 
A
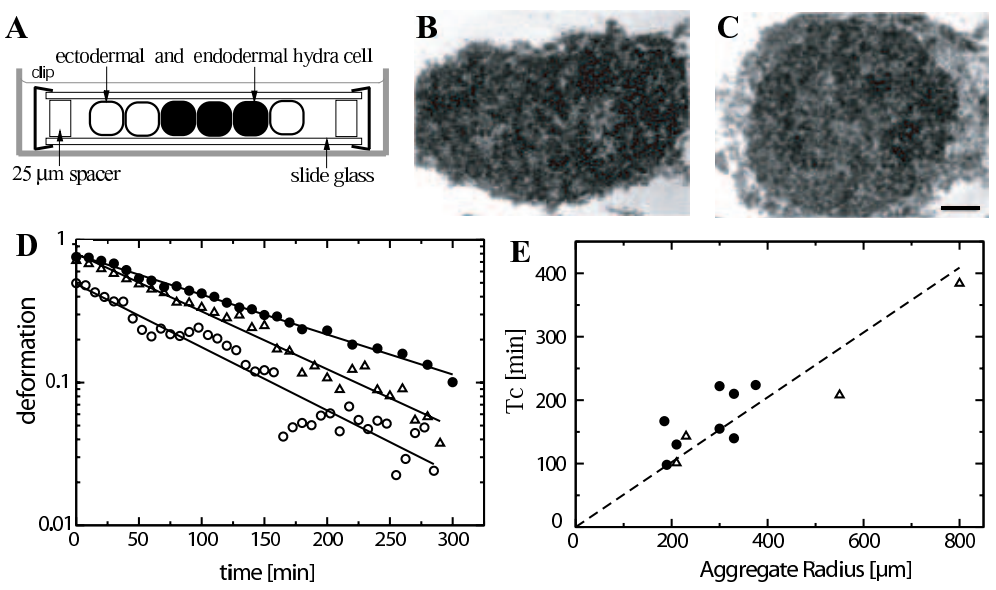

Figure 1: Shape relaxation in 2D Hydra aggregates (data from [8]). (A) 2D experimental set-up used to study aggregate shape relaxation. (B) A pure endodermal aggregate at $0 \mathrm{~h}$ and $(\mathrm{C})$ at $6 \mathrm{~h}$. Bar, $100 \mu \mathrm{m}$. (D) Deformation parameter $d$ (see text) as a function of time (bullets: pure endodermal aggregate; triangles: mixed aggregate; circles: pure ectodermal aggregate). (E) Relaxation time $T_{c}$ as a function of aggregate radius (same legend as D). 

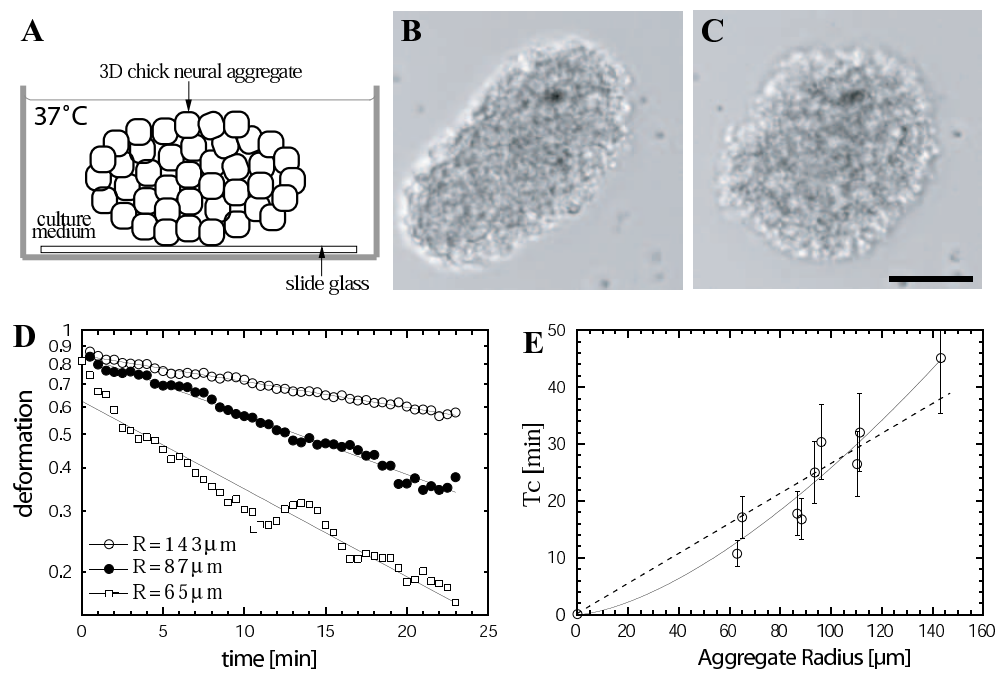

Figure 2: Rounding of 3D chicken embryonic neural retina aggregates. (A) Configuration used to study shape relaxation: the 3D aggregates are floating near to the bottom of glass slide in culture medium at $37^{\circ} \mathrm{C}$. (B) A small 9-day old neural retina aggregate $(\mathrm{R}=63 \mu \mathrm{m})$ at $0 \mathrm{~h}$ and $(\mathrm{C})$ at $24 \mathrm{~h}$. Bar, $50 \mu \mathrm{m}$. (D) Deformation parameter $d$ as a function of time for three different aggregate sizes. (E) Relaxation time $T_{c}$ as a function of aggregate radius. Solid line is a power law fit $\left(\sim R^{1.52}\right.$, correlation coefficient, 0.939$)$, dotted line is a linear fit with with Eq. 2 (correlation coefficient, 0.880). 

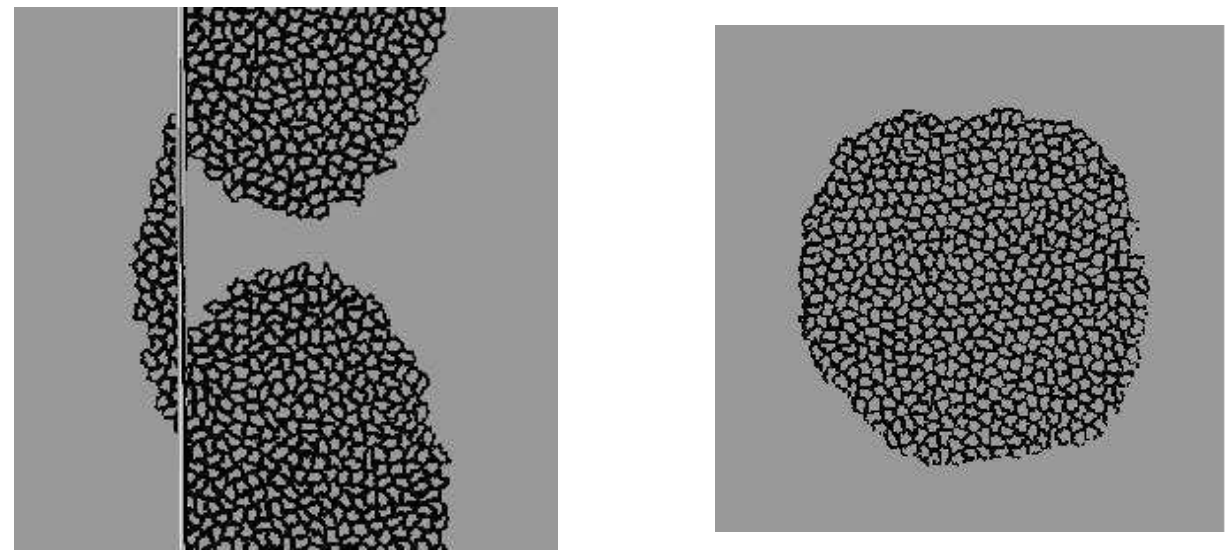

Figure 3: (A) Image of an initial elliptical aggregate with 544 cells. (B) Final state of the aggregate after, approximately, $10^{6}$ MCS. 


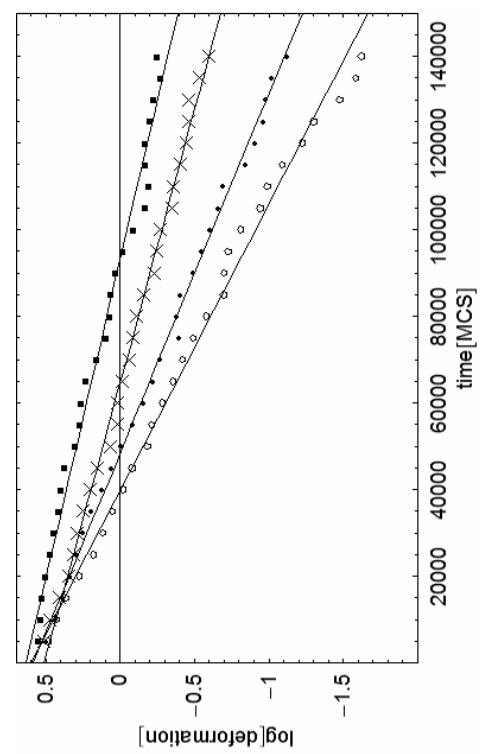

Figure 4: Log-linear plot of deformation as a function of time for simulated aggregates with 1642 cells and surface tensions 4.5 (squares), 6.5 (crosses), 8.5 (dots), and 9.5 (circles). 


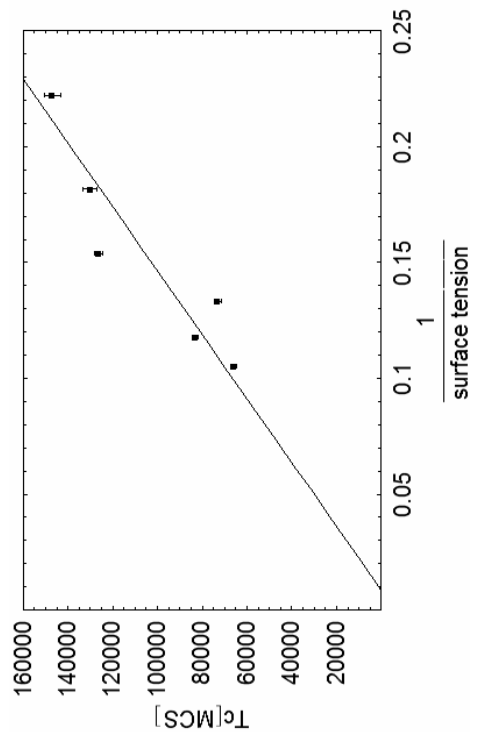

Figure 5: Plot of $T_{c}$ as a function of $\sigma^{-1}$ for simulated aggregates with 1642 cells and surface tensions $4.5,5.5,6.5,7.5,8.5$, and 9.5. The correlation coefficient of the fit is 0.85 . 


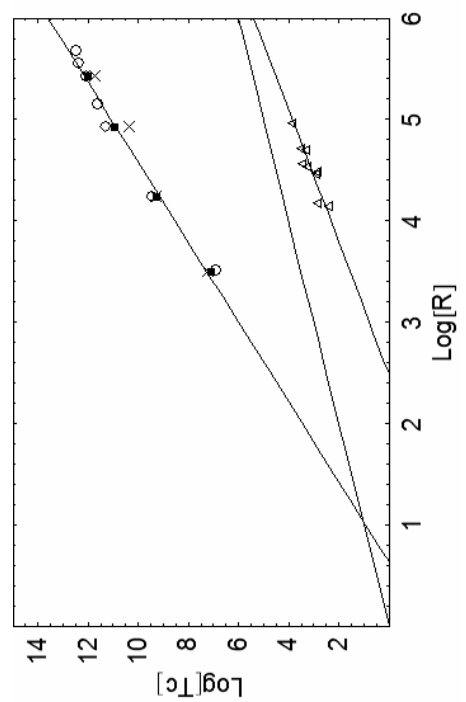

Figure 6: $\log -\log$ plot of $T_{c}$ vs. $R$ for simulation and chicken data (triangles). $R$ is lattice sites for simulation and $\mu m$ for experiments. Simulation surface tensions are: 4.5 (circles), 5.5 (squares), 6.5 (crosses). An identity line is shown for slope comparison. 